PAPER

\title{
Metacognitive deficits in frontotemporal dementia
}

\author{
P J Eslinger, K Dennis, P Moore, S Antani, R Hauck, M Grossman
}

J Neurol Neurosurg Psychiatry 2005;76:1630-1635. doi: 10.1136/jnnp.2004.053157

See end of article for authors' affiliations

.....................

Correspondence to:

Dr P J Eslinger, Department

of Neurology-H037, Penn

State Milton S Hershey

Medical Center, PO Box

850 , Hershey, PA 17033-

0850, USA; peslinger@

psu.edu

Received8September 2004

Revised version received

8 March 2005

Accepted 10 April 2005

\begin{abstract}
Objectives: To investigate whether metacognitive impairments in self-awareness and self-monitoring occur in patients with frontotemporal dementia (FTD), particularly among those with prominent social and dysexecutive impairments.

Methods: Patients diagnosed with FTD were divided by clinical subtype (social-dysexecutive $(n=12)$ aphasic $(n=15)$, and constituent subgroups of progressive non-fluent aphasia and semantic dementia) and compared with subjects with probable Alzheimer's disease $(A D, n=11)$ and age-matched healthy controls $(n=11)$. All subjects completed comprehensive behavioural ratings scales, which were compared with caregiver ratings. Subjects also rated their test performances in verbal associative fluency, word list learning, and memory task with comparisons made between actual and judged performance levels.

Results: The FTD sample as a whole showed significantly less behavioural self-awareness and selfknowledge than the $A D$ and healthy control samples. FTD patients with prominent social and dysexecutive impairments demonstrated the most extensive loss of self-awareness and self-knowledge, significantly overrating themselves in multiple social, emotional, and cognitive domains, and failing to acknowledge that any behavioural change had occurred in most areas. The remaining clinical samples showed select and minimal discrepancies. All clinical groups were significantly unaware of their apathy levels. Most FTD patients judged episodic cognitive test performance adequately, with partial difficulties observed in the socially impaired and progressive non-fluent aphasia subgroups.

Conclusions: FTD patients, particularly those with prominent social and dysexecutive impairments, exhibit profound metacognitive anosognosia that may represent a loss of self-awareness, self-monitoring, and self-knowledge, likely related to significant prefrontal pathophysiology. Other FTD clinical groups and AD patients showed less pervasive and more select metacognitive deficiencies.
\end{abstract}

$M$ tacognition refers to diverse processes of self-knowledge, self-monitoring, and self-regulation that contribute to adaptive behaviour in complex and changing environments. ${ }^{1-4}$ Metacognition is considered a domain of information processing that comprises personal awareness and knowledge as well as adaptive strategies. These processes can be operationalised as experimental measures in certain tasks. For example, individuals can be asked to predict their expected performance in a particular task, such as learning a word list. This entails derived selfknowledge of abilities (established on the basis of various previous experiences, incorporation of feedback, and selfreflection), experiences and synthesis of that information for judgement purposes. Individuals can also be asked how hard or easy it will be to learn particular words, as in judgement of learning measures that draw upon knowledge of stimulus characteristics and previous experience with those types of items. In addition to self-predictions, metacognition can be probed by securing judgements of how individuals actually performed after the task is completed. This facet emphasises a self-monitoring aspect of metacognition and permits comparisons of perceived versus actual performance. Metacognitive processing is also thought to contribute to self-regulation of behaviour through central executive functions. This includes how effectively and accurately an individual can use self-knowledge and self-monitoring abilities to guide cognition and behaviour in social and non-social contexts. ${ }^{5}$ Standardised behavioural scales provide the commonest approach to surveying these functions in real-world settings on a daily basis, often with confirmatory information from caregivers and family members.

In neurobehavioural studies, metacognition has been associated predominantly with prefrontal cortical systems through studies of patients with focal cerebral lesions. For example, prefrontal damage has been associated with overestimation of abilities, lack of awareness of deficits, inability to learn from experience as well as use feedback about behaviour, contributing to severe decision making and selfregulatory defects. These impairments are often described as forms of anosognosia that occur within problem solving and social-emotional contexts even though general cognitive abilities remain intact. ${ }^{6-12}$ Such findings suggest that clinical disorders of self-awareness and metacognition can be associated with prefrontal pathophysiology and lead to profound adaptive deficits in daily functioning. Certain lines of research have linked metacognitive deficits to impaired executive functions mediated principally through prefrontal cortical systems, ${ }^{13}$ supporting an interrelation between metacognition and executive functions. The other major presentation of self-awareness deficits is that associated with large right middle cerebral artery stroke causing profound hemispatial neglect as well as sensory-motor deficits. In this case, anosognosia is most prominent in sensory-motor and spatial attentional domains. ${ }^{14}$

Metacognition has been examined only minimally in patients with neurodegenerative disease. Although some studies have reported fairly accurate self-prediction and self-monitoring abilities of patients with Alzheimer's disease $(\mathrm{AD})$ in learning and memory tasks, ${ }^{15}{ }^{16}$ other studies have reported impaired awareness of memory deficits in AD. ${ }^{16-21}$ Barrett et $a l^{21}$ reported domain specific self-prediction (pretest) and self-monitoring (post-test) deficits when $\mathrm{AD}$ patients undertook tasks of visuospatial skill and memory, respectively. Prediction and evaluation of other domains such as naming, limb praxis, attention, and mood were

Abbreviations: $A D$, Alzheimer's disease; FTD, frontotemporal dementia; PNFA, progressive non-fluent aphasia; SD, senile dementia 
comparable with controls. In some reports, metamemory deficits in $\mathrm{AD}$ have been correlated with executive function impairments as well hypoperfusion of the right dorsolateral frontal region. ${ }^{22-24}$

Metacognition has not been studied empirically in patients with frontotemporal dementia (FTD). Among patients with FTD, those with prominent social and dysexecutive impairments commonly exhibit clinical disorders of self-awareness, self-regulation, and self-monitoring. ${ }^{25}$ It is suspected that these deficits contribute significantly to their progressive adaptive behavioural difficulties in home, vocational, and social settings, leading to disability and supervisory care. ${ }^{26}$ FTD patients with predominant frontal cortical pathology on brain magnetic resonance imaging have demonstrated deficits on executive function measures sensitive to frontal lobe lesions and develop significant social behavioural impairments that include loss of empathy, impaired judgement, disinhibition, and apathy. ${ }^{27-30}$ In patients with focal cortical lesions from other causes, alterations of empathy, judgement, self-regulation, self-awareness, and social adaptation have been linked principally to prefrontal damage involving medial and orbital regions. ${ }^{11} 1231$ Therefore, we hypothesised that FTD patients with clinically prominent social and dysexecutive impairments typically associated with prefrontal pathology would show measurable and disproportionate deficits in metacognition in comparison to FTD patients with clinically prominent aphasia and patients with $\mathrm{AD}$. We employed two approaches to measuring aspects of metacognition: (a) quantitative comparison of patient and caregiver appraisals of the patient's daily functioning on standardised behavioural inventories; and $(b)$ quantitative analysis of self-prediction and self-monitoring abilities on cognitive tasks. To compensate for reporter variation, we compared the results with a sample of healthy controls and the appraisals provided by their respective family members.

\section{METHODS}

\section{Subjects}

We recruited all patients for longitudinal study through clinical services with a diagnosis of probable $\mathrm{AD}^{32}$ and FTD ${ }^{33} 34$ based on standard criteria. All patients provided informed consent according to protocols approved by the Institutional Review Board of the Hospital of the University of Pennsylvania. Characteristics of the samples are summarised in table 1.

We analysed the FTD sample $(n=27)$ as a whole and also divided the patients into subgroups according to a consensus based clinical assignment by two independent examiners. We drew criteria from prominent clinical and behavioural characteristics. ${ }^{25}$ 35-38 The FTD subgroups included nonaphasic and aphasic patients. The non-aphasic patients demonstrated predominantly social behavioural and dysexecutive impairments (SOC-DYSEX) $(n=12)$. The aphasic patients were identified as a single group (APHASIC) $(\mathrm{n}=15)$ and also further divided into those with features of either progressive non-fluent aphasia (PNFA) or semantic dementia (SD). All clinical participants were alert, cooperative, and able to complete all tasks presented to them.

We recruited the older healthy control participants from available volunteer resources resulting from local advertisements.

\section{Procedures}

\section{Behavioural rating scales}

The subjects and their respective caregivers completed a comprehensive series of behaviour rating inventories to survey a broad range of everyday cognitive, social, and emotional behaviours. The modified Brock Adaptive Functioning Inventory ${ }^{39}$ contains 40 items that query about daily functional capacities in planning, initiation, cognitive flexibility, excessive caution, attention, memory, motivation/ arousal, emotionality, impulsivity, aggression, self-monitoring, and empathy. Ratings are standardised along a numeric scale, providing a quantitative score in each domain. Respondents also indicated whether there has been a change in behavioural ability for each item relative to the patient's natural baseline (that is, same-different analysis). The Apathy Evaluation Scale ${ }^{40}$ contains 18 items designed to assess cognitive, behavioural, and emotional aspects of goal directed behaviour. The scale is particularly sensitive to loss of motivation not attributable to altered consciousness, cognition, or emotional distress. The final instrument that we used was the Interpersonal Reactivity Index, which is designed to assess multiple dimensions of empathy. ${ }^{41}$ There are four seven-item subscales that tap perspective taking (more cognitively oriented), empathic concern (more affectively oriented), personal distress (emotional oversensitivity to others), and fantasy (ability to imagine oneself in fictional situations). In each behavioural domain, ratings were normalised to range from 0 to 100 , providing a basis for comparison across domains and scales. On each item (including both behavioural ratings and ratings of any change), we subtracted the caregiver rating from the respective self-rating of the subject, obtaining a discrepancy score that ranged from negative (caregiver rating the subject as more capable and unchanged in comparison with the subject's self-rating) to positive (caregiver rating the subject as less capable and changed from baseline in comparison with the subject's self-rating).

\section{Cognitive tasks}

The subjects quantitatively predicted and evaluated their cognitive test performance that was then compared with their actual test scores. The standardised cognitive measures included: verbal associative fluency, word list learning, and verbal memory.

In the verbal associative fluency task, we asked the subjects to generate as many animal names as possible within one minute. Prior to performing the task, they indicated how easy or hard the task would be by marking

Table 1 Characteristics of frontotemporal dementia (FTD total; social-dysexecutive subtype; aphasic subtype; progressive non-fluent aphasia subtype (PNFA); semantic dementia (SD) subtype), Alzheimer's disease (AD), and healthy control samples

\begin{tabular}{llllllll}
\hline & Total FTD & $\begin{array}{l}\text { Social- } \\
\text { dysexecutive }\end{array}$ & Aphasic & PNFA & SD & AD & Control \\
\hline No of subjects & 27 & 12 & 15 & 7 & 8 & 11 & 11 \\
Mean age (range) & 63.64 & 61.63 & 68.07 & 69.00 & 67.14 & 73.09 & 75.09 \\
& $(44-82)$ & $(41-76)$ & $(55-82)$ & $(55-82)$ & $(55-75)$ & $(51-81)$ & $(64-83)$ \\
Mean education & 14.71 & 15.30 & 14.13 & 14.00 & 14.25 & 14.78 & 14.64 \\
MMSE score & 23.45 & 24.42 & 22.67 & 22.14 & 23.13 & 23.36 & 29.36 \\
\hline
\end{tabular}

MMSE, Mini-Mental State Examination. 
an $X$ along a visual analogue scale consisting of a $20 \mathrm{~cm}$ vertical line on a $21.6 \times 27.9 \mathrm{~cm}$ sheet of paper. ${ }^{40}$ The top of the line was marked by the words CAN SAY MANY WORDS and the bottom was marked by CAN SAY NO WORDS AT ALL. At $5 \mathrm{~cm}$ markers, simple face drawings indicated either happiness (at top), neutral (at middle) or sadness (at bottom) to provide a non-verbal cue in rating their expected performance. Immediately following the task, subjects similarly rated how well they performed, using the same scaling procedure with the top now marked by the words VERY HIGH TEST SCORE and the bottom by VERY LOW TEST SCORE. We converted the subject ratings on the visual analogue scale to a proportional quantitative score varying between 0 (very low) and 100 (very high).

In the verbal list learning task, subjects were instructed that they would be read 10 words and they would have to recall all 10 or as many as they could remember. Before actual testing, the subjects indicated how easy or hard it would be to learn 10 words by placing an $\mathrm{X}$ along a visual analogue scale, similar to the one described above but with the caption CAN LEARN ALL 10 WORDS at the top and NOT ABLE TO LEARN ANY WORDS AT ALL at the bottom. They then proceeded to three learning trials of the 10 word list taken from the Consortium to Establish a Registry for Alzheimer's Disease (CERAD) battery. ${ }^{42}$ Immediately following the third trial, subjects rated how well they performed by placing an $\mathrm{X}$ along the visual analogue scale with the captions VERY HIGH LEARNING TEST SCORE at the top and VERY LOW LEARNING TEST SCORE at the bottom.

Following 10 minutes of distraction tasks, subjects were enlisted to recall as many of the 10 words as possible for the verbal memory measure. They first predicted their performance along the same visual analogue scale used previously and then rated their actual performance in a similar fashion.

\section{RESULTS}

Comparisons of samples on background measures indicated that the mean age of SOC-DYSEX group was younger than that of the controls and AD patients $(\mathrm{p}<0.001)$. Patients with FTD-aphasia were also younger than the controls $(p<0.05)$ but there were no other age differences. The groups did not differ according to educational background. Analysis showed significant group differences in Mini-Mental State Examination (MMSE) scores with all patient groups scoring significantly lower than the control group $(\mathrm{p}<0.01)$ but not different from each other. Despite their language impairments, aphasic patients did not differ from non-aphasic patients in the cognitive screening score.

\section{Rating scales of cognition, behaviour, emotion, and empathy}

Each subject provided comprehensive quantitative ratings of their everyday cognition, behaviour, emotion, and empathy that were then compared with their own caregiver's or close family member's ratings. Any discrepancy between subject and caregiver ratings was then computed for each behavioural domain and compared with those generated by healthy controls and their caregivers/family members. This approach, therefore, took into account the naturally occurring discrepancy between research participants and their caregivers/family members, and only discrepancy scores that were statistically different from control levels $(p<0.05)$ were identified. The mean scores and their discrepancy for each behavioural domain are summarised in table 2 .

Multivariate analysis of variance indicated a significant interaction effect $(\mathrm{p}<0.05)$, with post hoc tests confirming that the FTD sample as a whole consistently overestimated their abilities in eight of 17 domains (47\%). This was four times the level of the AD sample (12\%). Analysis of the FTD subtypes indicated that these differences were accounted for mainly by the SOC-DYSEX sample who were significantly discrepant in 10 of 17 domains (59\%). This high level of discrepancy was in marked contrast to the FTD-aphasia subtype (12\%) and its constituent PNFA (6\%) and SD (18\%) samples $(\mathrm{p}<0.01)$. Significant differences were evident for the domains of planning, cognitive flexibility, self-monitoring,

Table 2 Behaviour ratings of subjects with frontotemporal dementia (FTD) versus caregivers across 17 domains in comparison with Alzheimer's disease (AD) and control samples. Differences between subjects' and caregivers' scores are in parentheses, with positive values indicating that subjects rated themselves with comparatively more capability in the behaviours domain than their caregivers

\begin{tabular}{|c|c|c|c|c|c|c|c|}
\hline & \multicolumn{5}{|c|}{ Frontotemporal dementia } & \multicolumn{2}{|l|}{ Comparison groups } \\
\hline & \multirow{2}{*}{$\begin{array}{l}\text { Total sample } \\
\text { Self-Caregiver }\end{array}$} & \multirow{2}{*}{$\begin{array}{l}\begin{array}{l}\text { Social- } \\
\text { behavioural }\end{array} \\
\text { Self-Caregiver }\end{array}$} & \multirow{2}{*}{$\begin{array}{l}\text { APHASIC (PNFA } \\
\text { and SD) } \\
\text { Self-Caregiver }\end{array}$} & \multirow{2}{*}{$\begin{array}{l}\text { PNFA } \\
\text { Self-Caregiver }\end{array}$} & \multirow{2}{*}{$\frac{\text { SD }}{\text { Self-Caregiver }}$} & \multirow{2}{*}{$\frac{A D}{\text { Self-Caregiver }}$} & \multirow{2}{*}{$\frac{\text { Controls }}{\text { Self-Caregiver }}$} \\
\hline & & & & & & & \\
\hline \multicolumn{8}{|l|}{ Brock scale } \\
\hline Impulsivity & $71.7-67.4(4.3)$ & $68.8-55(13.8)$ & $73.8-76.4(-2.6)$ & $74.5-90(-15.5)$ & $73.3-65(8.3)$ & $80-77(3)$ & $77.8-78.9(-1.1)$ \\
\hline Aggression & $86.9-81.4(5.5)$ & $79-74(5)$ & $92.7-86.8(5.9)$ & $92-90(2)$ & $93.3-84.2(9.1)$ & $87-93(-6)$ & $90.5-89.4(1.1)$ \\
\hline Self-monitoring & $66.8-48.2(18.6)^{*}$ & $64.5-40.7(23.8)^{*}$ & $68.5-53.6(14.9)$ & $66.8-58(8.8)$ & $70-50(20)^{*}$ & $70-67(3)$ & $76.1-76.1(0)$ \\
\hline Empathy & $77.9-56.8(21.1)^{*}$ & $78.1-40.5(37.6)^{*}$ & $77.7-68.6(9.1)$ & $77.5-67(10.5)$ & $77.8-69.9(7.9)$ & $93.3-91.1(2.2)$ & $84.4-77.9(6.5)$ \\
\hline Emotionality & $76.4-75.8(0.6)$ & $71.9-70(1.9)$ & $79.6-80(-0.4)$ & $80-86(-6)$ & $79.2-75(4.2)$ & $90-80(10)$ & $89.4-83.9(5.5)$ \\
\hline Aftention & $68.4-48.9(19.5)^{*}$ & $70-42.5(27.5)^{*}$ & $67.2-53.6(13.6)$ & $65.9-54(11.9)$ & $68.3-53.3(15)$ & $78.3-60(18.3)$ & $79.4-81(-1.6)$ \\
\hline Memory & $74.7-57.8(16.9)$ & $81.2-50.6(30.6)^{*}$ & $69.9-63(6.9)$ & $71.8-64.5(3.2)$ & $68.3-61.7(6.6)$ & $80-50(30)^{*}$ & $81.1-85.3(-4.2)$ \\
\hline Arousal & $70.5-53.9(16.6)^{*}$ & $66.3-35.1(31.2)^{*}$ & $73.5-67.5(6)$ & $71.8-64.5(6.3)$ & $75-70(5)$ & $86.7-73.3(13.4)$ & $78.9-75.6(3.3)$ \\
\hline Planning & $75.2-53.6(21.6)^{*}$ & $73.7-38.7(35)^{*}$ & $76.3-64.5(11.8)$ & $74-64(10)$ & $78.3-65(13.3)$ & $71.7-60(11.7)$ & $89.4-85(4.4)$ \\
\hline Initiation & $71.2-59.6(11.6)$ & $76.6-48.3(28.3)^{*}$ & $67.3-67.9(-0.6)$ & $58.7-68(-9.3)$ & $74.4-67.8(6.6)$ & $82.2-62.2(20)$ & $83.7-81.4(2.3)$ \\
\hline Flexibility & $70.6-44.9(25.7)$ & $77.5-35(42.5)^{*}$ & $65.5-52.1$ (13.4) & $66.7-53(13.4)$ & $64.5-51.1(13.4)$ & $77.8-68.9(8.9)$ & $82.9-77.7(5.2)$ \\
\hline Excess caution & $62.4-52.6(9.8)$ & $56.6-38.3(18.3)$ & $66.7-63(3.7)$ & $68-81.3(-13.3)$ & $65.6-47.8(17.8)$ & $84.4-51.1$ (33.3) & $70.3-71.1(-0.8)$ \\
\hline Apathy scale & $81.1-57.1(24)^{\star}$ & $82.5-41.4(41.1)^{*}$ & $80.1-68.6(11.5)^{*}$ & $77-68.9(8.1)^{*}$ & $82.6-68.3(14.3)^{*}$ & $87.9-67.6(20.3)^{*}$ & $77-82.7(-5.7)$ \\
\hline \multicolumn{8}{|l|}{ Empathy scale } \\
\hline Fantasy & $32.4-21.2(11.2)$ & $28.9-20.4(8.5)$ & $35-21.8(13.2)$ & $31.4-25.7(5.7)$ & $38-18.6(19.4)$ & $26.7-20(6.7)$ & $35.2-30.8(4.4)$ \\
\hline Perspective taking & $47.3-25.3(22)^{*}$ & $49-19.8(29.2)^{*}$ & $46.2-29.3(16.9)$ & $46.3-33.6$ (12.7) & $46.2-25.7(20.5)^{*}$ & $50.5-41.9(8.6)$ & $46.4-43.8(2.6)$ \\
\hline Empathic concern & $43.5-34.7(8.8)$ & $41.6-33.8(7.8)$ & $44.9-35.3(9.6)^{*}$ & $45.1-37.2(7.9)$ & $44.8-33.8(11)^{*}$ & $35.2-47.6(-12.4)$ & $42.2-46.1(-3.9)$ \\
\hline Personal distress & $39.6-25.9(13.7)^{*}$ & $35.1-23.5(11.6)$ & $42.9-27.7$ (15.2) & $40.6-27.2(13.4)$ & $44.8-28.1(16.7)$ & $21.9-30.5(-8.6)$ & $36.8-35.2(1.6)$ \\
\hline $\begin{array}{l}\text { No of discrepant } \\
\text { domains }\end{array}$ & 8 & 10 & 2 & 1 & 3 & 2 & - \\
\hline
\end{tabular}


initiation, motivation/arousal, attention, memory, empathy, perspective taking aspects of empathy, and apathy. The other neurological samples exhibited only selective discrepancies. These included: apathy in all the clinical samples, suggesting that they viewed themselves as much more motivated and vital than their caregivers did; empathic concern in the overall aphasic subtype; selfmonitoring and empathic concern in the SD subtype; and memory in the $\mathrm{AD}$ sample. The domains of excessive caution in the $\mathrm{AD}$ sample and perspective taking ability in the SD sample showed a statistical trend towards similar impairment. The SOC-DYSEX sample was clearly the most discrepant among clinical samples. This was reflected not only in the high number of affected behavioural domains but also by their elevated average discrepancy score in comparison with the other groups (fig 1).

When queried about whether the subjects exhibited any change in behaviour from their natural baseline (samedifferent analysis) on the modified Brock inventory items, a similar pattern of findings emerged. The SOC-DYSEX sample was significantly different from controls $(p<0.001)$, failing to acknowledge behavioural change observed by caregivers in 10 of the 12 Brock scales (83\%), in comparison with six of 12 scales for the FTD-aphasic sample $(50 \%)$ and similar levels for the PNFA (42\%) and SD (42\%) subtypes. The AD sample showed no significant differences from controls. Thus, all FTD samples showed some failure to acknowledge behavioural change, but this was most frequent in the SOCDYSEX subtype $(\mathrm{p}<0.05)$.

\section{Self-awareness of cognitive test performance}

Although pretest and post-test ratings were collected for each cognitive measure, we found the pretest ratings to be highly variable with few significant correlations to actual test performance, even among controls subjects. Thus, we chose not to examine those data any further and focused on posttest ratings as the clearest indication of subjects' direct awareness and monitoring of their cognitive abilities. Analysis of subjects' actual scores on the cognitive tests showed a significant group difference on each test $(\mathrm{p}<0.001)$. With regard to actual versus judged performance, results indicated that the total FTD sample showed significant positive correlations, similar to healthy controls (table 3). This suggested that post-test ratings generally varied in accordance with actual performance levels. This pattern held up fairly well in the FTD-aphasic sample and its SD subgroup.

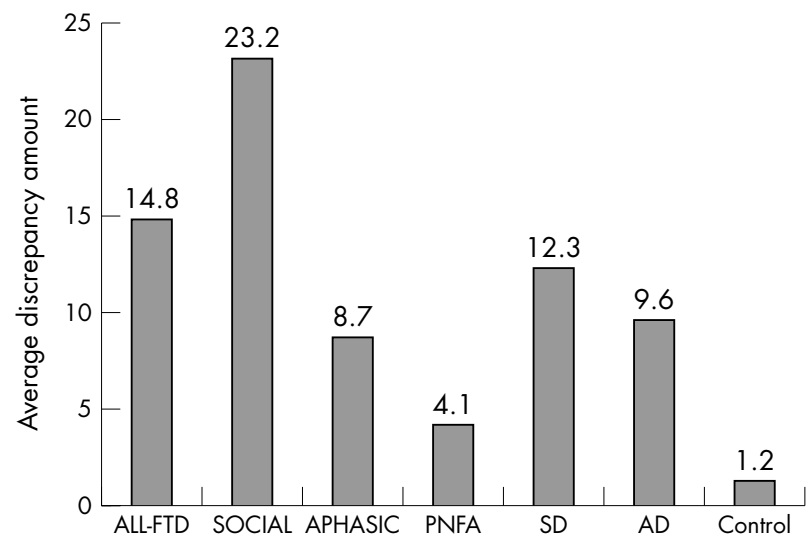

Figure 1 Degree of behavioural rating discrepancies across 17 behavioural domains for frontotemporal dementia (FTD) and healthy controls. AD, Alzheimer's disease; ALL FTD, total FTD sample; APHASIC, aphasic subtype; PNFA, progressive non-fluent aphasia subtype; SD, semantic dementia subtype; SOCIAL, social and dysexecutive subtype.
Table 3 Correlations of the subjects' post-test ratings of their performance with actual cognitive test scores

\begin{tabular}{|c|c|c|c|}
\hline & \multicolumn{3}{|l|}{ Post-test } \\
\hline & Verbal fluency & Word learning & Word memory \\
\hline \multicolumn{3}{|l|}{ FTD subgroups } & $0.537^{* *}$ \\
\hline SOC-DYSEX & $0.599^{* *}$ & $0.461^{*}$ & 0.368 \\
\hline APHASIC & $0.694^{* *}$ & $0.798^{* *}$ & $0.735^{\star *}$ \\
\hline PNFA & 0.308 & $0.858^{\star *}$ & $0.802^{* *}$ \\
\hline SD & $0.884^{* *}$ & $0.602^{*}$ & $0.767^{* *}$ \\
\hline Alzheimer's disease & $0.512^{*}$ & $0.676^{* *}$ & $0.749^{* *}$ \\
\hline Controls & $0.821^{\star *}$ & $0.455^{\star}$ & $0.597^{\star *}$ \\
\hline \multicolumn{4}{|c|}{$\begin{array}{l}{ }^{*} 0.05>\mathrm{p}<0.10 \\
{ }^{* *} \mathrm{p}<0.05 \text {. } \\
\text { APHASIC, aphasic subtype; FTD, frontotemporal dementia; PNFA, } \\
\text { progressive non-fluent aphasia; SD, senile dementia; SOC-DYSEX, social } \\
\text { behavioural and dysexecutive subtype. }\end{array}$} \\
\hline
\end{tabular}

The SOC-DYSEX and PNFA samples each showed selective difficulty in judging performance on one task (word memory for the SOC-DYSEX sample and verbal fluency for the PNFA sample). These were the only samples with subjects who did not decrease their pretest rating after performing below the mean for their sample and for all neurological subjects ( $1 / 7$ PNFA subjects and 2/9 SOC-DYSEX subjects). The AD sample showed levels comparable with controls and the total FTD group.

\section{DISCUSSION}

We examined self-awareness, self-monitoring, and selfknowledge aspects of metacognition in patients with FTD using quantitative measures of their behavioural capacities as well as cognition. Self-judgements were compared with either the judgements of a close caregiver or to actual cognitive test scores. With regard to patient-caregiver comparisons, we took into account the natural variations that occur in behavioural ratings by identifying only those discrepancies that statistically exceeded those measured between healthy controls and their close caregivers, ensuring a more conservative analysis.

\section{Performance on the rating scales}

In comparison with the $\mathrm{AD}$ and healthy control samples, patients with FTD showed significantly less self-awareness and self-knowledge of their daily behavioural abilities and deficits, even though they were somewhat younger. This was most evident in domains of arousal, attention, self-monitoring, planning, and empathy (including perspective taking and personal distress) and in rating of their apathy levels. Patients with FTD fared comparatively better when they were asked to judge performance on a specific cognitive task they had just completed, showing significant positive correlations to actual test scores. The difference between the two patterns of results may be related to the specific, episodic nature of the cognitive testing as opposed to more general patterns of their day to day functioning that may require more inferential processing and perspective taking. Patients with FTD appeared to vary widely in their metacognitive abilities, and our study design allowed analysis of multiple clinical subtypes.

FTD SOC-DYSEX patients with clinically prominent social and dysexecutive deficits were the most consistently and severely impaired in appreciating their everyday behavioural deficits. This was apparent in both quantitative ratings of their behavioural capacities and their acknowledgement of whether any change had indeed occurred. Their loss of awareness and knowledge was evident in multiple behavioural domains, spanning social-emotional functioning 
(empathy, perspective taking, self-monitoring), motivation (arousal, initiation, apathy), and cognition (attention, memory, planning, flexibility). This profile of statistically significant differences from healthy controls suggests that impaired self-awareness and self-knowledge are pervasive deficits affecting the metacognitive capacities of FTD patients with prominent social and dysexecutive impairments. These data are consistent not only with clinical characterisations of this subgroup ${ }^{25-27}$ but also suggest that metacognitive impairments may be important contributing factors to their social behavioural impairments of disinhibition, erratic judgement, poor self-monitoring, and declining adjustment to progressive deficits. In contrast, the other FTD clinical subgroups and the $\mathrm{AD}$ sample showed metacognitive loss in only a few, select behavioural domains, suggesting a minimal deficit. The FTD-aphasic sample overall showed statistically significant discrepancies just in apathy and empathic concern. The large degree of agreement between aphasic participants and their caregivers provides further confidence in the accuracy of their language processing in these particular tasks. Among the aphasic subjects, the FTD-SD sample was significantly impaired in awareness of changes in self-monitoring, apathy and to a modest degree in perspective taking and emotional concern aspects of empathy. In contrast, the FTD-PNFA sample was different from the healthy controls only in their limited awareness of apathetic changes, though this was a modest difference, the least among the clinical groups. The AD sample was significantly impaired in awareness of memory and apathy changes only.

Our findings regarding empathy are particularly noteworthy. The FTD SOC-DYSEX sample was not only rated by caregivers with the lowest average scores of all the samples, but also showed the widest discrepancy from caregiver ratings. This was evident from the three question empathy domain of the Brock Scale and on the perspective taking dimension of empathy that encompasses more cognitively oriented processes of perceiving and considering another's viewpoint or situation. These patients' lack of awareness of empathic changes can be associated with significant interpersonal problems, as their view of themselves in relationship to others can differ dramatically from family members and caregivers. The only other FTD sample to show any empathy related discrepancies was the SD, with comparatively greater differences from caregiver ratings in perspective taking and empathic concern. The $\mathrm{AD}$ sample showed no significant discrepancies in empathic ratings.

\section{Self-awareness of cognitive test performance}

With regard to judging their cognitive test performances, we focused on the subjects' post-test evaluations as the clearest indication of cognitive self-awareness. The pretest predictions of their cognitive test performance were difficult to interpret because subjects might well have had differing conceptions of what the test would entail and how easy or difficult it would be. In the analysis of post-test ratings of performance, FTD patients fared comparatively well, as only select difficulties were demonstrated. The FTD TOTAL, APHASIA, and SD samples as well as $\mathrm{AD}$ and healthy controls all showed significant positive correlations between actual test performance and judged performance on all three tasks. The FTD SOC-DYSEX patients were the only ones to poorly judge their learning performance and the FTD PNFA patients were the only ones to poorly judge their verbal associative fluency performance, but they were otherwise comparable with the controls.

\section{Rating scales results versus self-judgement}

The relative differences between the behavioural rating scale results and the judgement of test performance may have emanated from the fact that the judged test performance was immediately experienced and more straightforward with regard to its parameters (for example, number of words). Thus, FTD patients may be able to show accurate selfjudgements under certain circumstances that are highly structured and well focused, essentially demanding fewer cognitive resources. In contrast, ratings of daily functioning and behavioural change were related to less specific situations and drew upon broader domains of self-knowledge and experiences across multiple settings and time frames. Such processing likely required greater utilisation of self-knowledge domains through synthesis of varied experiences over time, and drew upon more cognitive resources. In this sense, the deficits of self-awareness and self-monitoring in FTD, particularly among the FTD SOC-DYSEX subtype, correspond to a loss of self-knowledge as a representational domain. ${ }^{43}$ This impairment may become most evident in situations that necessitate more cognitive resources, compounding the loss of actual behavioural and emotional abilities in FTD. ${ }^{25}$ The pattern of metacognitive anosognosia appeared to separate FTD patients from $\mathrm{AD}$ patients as well as healthy controls. Furthermore, the patients with FTD SOC-DYSEX subtype showed far greater metacognitive anosognosia than those with the APHASIC subtype, including PNFA and SD, who may develop social-emotional changes but without the metacognitive component ${ }^{4445}$

\section{Further considerations}

During data analysis, we informally observed a further trend; namely that there may be another subtype of FTD within the SOC-DYSEX sample. These are patients who present with primarily dysexecutive impairments that affect planning, organisation, cognitive flexibility and self-monitoring, but without the profound social-emotional and metacognitive deficits. This clinical pattern is reminiscent of focal lesions to the dorsolateral prefrontal region. ${ }^{46}$ We identified three such patients within this cohort. We suspect that a larger sample of patients with FTD may reveal additional cases that could eventually be analysed separately. Moreover, in future studies we plan to analyse these behavioural findings in relation to structural measures of brain atrophy so as to identify specific neural correlates and patterns. In the case of the SOC-DYSEX sample, we suspect that their metacognitive deficits will be related to prefrontal atrophy that affects primarily medial and orbital regions as described in the focal brain lesion literature. ${ }^{46}$

\section{CONCLUSION}

Self-awareness and self-knowledge are important to adaptive social-emotional functioning because of the highly interactive nature of human behaviour. Self-awareness entails continuing perception of our behaviour, self-monitoring of its causes and effects, synthesis of experiences over time and space, and a working sense of self that is both historical and contemporary. The FTD patients in this study showed significant self-awareness and self-knowledge deficits across a variety of behavioural domains in comparison to patients with $\mathrm{AD}$ and healthy controls. Furthermore, FTD patients with progressive and clinically prominent social and dysexecutive impairments (FTD SOC-DYSEX) demonstrated the poorest self-awareness and self-knowledge of FTD subjects across social, emotional, and cognitive domains. This would suggest that an important underlying deficit is their loss of adaptive metacognitive resources. Further studies are needed to identify the specific cognitive and/or emotional processing correlates of this profound anosognosia by examining executive functioning, theory of mind, autonomic reactivity, and other processing functions in FTD. In addition, morphometric brain analyses are needed to investigate the neuroanatomical bases and pathophysiology of metacognitive anosognosia. 


\section{Authors' affiliations}

P J Eslinger, Departments of Neurology and Neural and Behavioral Sciences, College of Medicine, Pennsylvania State University, Milton S Hershey Medical Center, Hershey, PA, USA

K Dennis, P Moore, S Antani, R Hauck, M Grossman, Department of Neurology, University of Pennsylvania School of Medicine, Hospital of the University of Pennsylvania, Philadelphia, PA, USA

This work was supported in part by the US Public Health Service (AG15116, NS44266, AG17586) and the American Health Foundation.

Competing interests: none declared

\section{REFERENCES}

1 Brown AL. Knowing when, where, and how to remember: a problem of metacognition. In: Glaser R, eds. Advances in Instructional Psychology. Hillsdale NJ: Erlbaum Associates, 1978:367-406.

2 Flavell J. Metacognition and cognitive monitoring: a new area of cognitivedevelopmental inquiry. Am Psychol 1979;34:906-11.

3 Metcalfe J, Shimamura AP. Metacognition: Knowing about Knowing. Cambridge MA: MIT Press, 1996.

4 Mazzoni G, Nelson TO. Metacognition and Cognitive Neuropsychology. Mahwah NJ: Erlbaum Associates, 1998.

5 Fernandez-Duque D, Baird J, Posner MI. Executive attention and metacognitive regulation. Conscious Cogn 2000;9:288-307.

6 Janowsky JS, Shumimura AP, Squire LR. Memory and metamemory: comparison between patients with frontal lobe lesions and amnesic patients. Psychobiology 1989;17:3-11.

7 Shimamura AP, Squire LR. Memory and metamemory: a study of feeling-ofknowing phenomenon in amnesic patients. J Exp Psychol: Learn Mem Cogn 1986; 12:452-60

8 Vilkki J, Servo A, Surma-Aho O. Word list learning and prediction of recall after frontal lobe lesions. Neuropsychology 1998;12:268-77.

9 Hanten G, Bartha M, Levin HS. Metacognition following pediatric traumatic brain injury: a preliminary study. Dev Neuropsychol 2000;18:383-98.

10 Eslinger PJ, Damasio AR. Severe disturbance of higher cognition after bilateral frontal lobe ablation: patient EVR. Neurology 1985;35:1731-41.

11 Dimitrov M, Phipps M, Zahn TP, et al. A thoroughly modern Gage. Neurocase 1999:5:345-54.

12 Eslinger PJ, Flaherty-Craig CV, Benton AL. Developmental outcomes after early prefrontal cortex damage. Brain Cogn 2004;55:84-103.

13 Dennis M, Barnes MA, Donnelly RE, et al. Appraising and managing knowledge: metacognitive skills after childhood head injury. Dev Neuropsychol 1996;12:77-103.

14 Clarke S. Right hemisphere syndromes. In: Bogousslavsky J, Caplan LR, eds. In:Stroke Syndromes, 2nd edn. Cambridge UK: Cambridge University Press, 2001:267-72.

15 Gil N, Josman N. Memory and metamemory performance in Alzheimer's disease and healthy elderly: the contextual memory test (CMT). Aging Clin Exp Res 2001;13:309-15.

16 Moulin CJ, Perfect TJ, Joues RW. Evidence for intact memory monitoring in Alzheimer's disease: metamemory sensitivity at encoding. Neuropsychologia 2000;38:1242-50.

17 McGlynn S, Kaszniak A. When metacognition fails: impaired awareness of deficits in Alzheimer's disease. J Cogn Neurosci 1991;3:183-9.

18 Green J, Goldstein F, Sirockinan B, et al. Variable awareness of deficits in Alzheimer's disease. Neuropsychiatry Neuropsychol Behav Neurol, 1993;6:159-65.

19 Lin KN, Teng EL, Wang PN, et al. Patients' versus caregivers' report of poor memory in relation to dementia and tested abilities. Neurology 2000;55:1758-9.

20 Duke LM, Seltzer B, Seltzer JE, et al. Cognitive components of deficit awareness in Alzheimer's disease. Neuropsychology 2002;16:359-69.
21 Barrett AM, Eslinger PJ, Ballentine NH, et al. Unawareness of cognitive deficit (cognitive anosognosia) in subjects with probable Alzheimer disease and matched controls. Neurology, (in press).

22 Reed BR, Jaquist WJ, Coueter L. Anosognosia in Alzheimer's disease; Relationship to depression, cognitive function and cerebral perfusion. J Clin Exp Neuropsychol 1994;15:231-44.

23 Lopez OL, Becker JT, Somsak D, et al. Awareness of cognitive deficits and anosognosia in probable Alzheimer's disease. Eur Neurol, 1994;34:277-82.

24 Michon A, Deweer B, Pillon B, et al. Relation of anosognosia to frontal lobe dysfunction in Alzheimer's disease. J Neurol Neurosurg Psychiatry 1993;57:231-44

25 Grossman M. Frontotemporal dementia: a review. J Int Neuropsychol Soc 2002;8:564-83.

26 Gregory CA, Hodges JR. Frontotemporal dementia: use of consensus criteria and prevalence of psychiatric features. Neuropsychiatry Neuropsychol Behav Neurol 1996;9:145-53.

27 Miller BL, Cummings JL, Villanueva-Meyer J, et al. Frontal lobe degeneration: clinical, neuropsychological and SPECT characteristics. Neurology 1991;54:2277-84.

28 Perry RJ, Rosen HR, Kramer JH, et al. Hemispheric dominance for emotions, empathy and social behaviour: evidence from right and left handers with frontotemporal dementia. Neurocase 2001;7:45-60.

29 Lindau M, Almkvist O, Kushi J, et al. First symptoms-frontotemporal dementia vs Alzheimer's disease. Dementia Geriatr Cogn Disord 2000;11:286-93.

30 Kramer JH, Jurik J, Sha SJ, et al. Distinctive neuropsychological patterns in frontotemporal dementia, semantic dementia, and Alzheimer disease. Cogn Behav Neurol 2003;16:211-18.

31 Eslinger PJ. Neurological and neuropsychological bases of empathy. Eur Neurol 1998;39:193-9.

32 McKhann G, Drachman P, Folstein M, et al. Clinical diagnosis of Alzheimer's disease: Report of the NINCDS-ADRDA work group under the auspices of the Department of Health and Human Services Task Force on Alzheimer's disease. Neurology 1984;34:939-44.

33 The Manchester and Lund Groups. Clinical and neuropathological criteria for frontotemporal dementia. J Neurol Neurosurg Psychiatry 1994;57:416-18.

34 McKhann G, Trojanowsji JQ, Grossman M, et al. Clinical and pathological diagnosis of frontotemporal dementia: report of a work group on frontotemporal dementia and Pick's disease. Arch Neurol 2001;58:1803-9.

35 Davis KL, Price C, Moore P, et al. Evaluating the clinical diagnosis of frontotemporal degeneration: A re-examination of Neary et al. 1998. Neurology 2001;56:A144-A145.

36 Price C, Davis KL, Moore P, et al. Clinical diagnosis of frontotemporal dementia (FTD). Neurology 2001;56:A176.

37 Neary D, Snowden JS, Gustafson L, et al. Frontotemporal lobar degeneration: a consensus on clinical diagnostic criteria. Neurology 1998;51:1546-54

38 Grossman M, Ash S. Primary progressive aphasia: a review. Neurocase 2004;10:3-18.

39 Dywan J, Roden R, Murphy T. Orbitofrontal symptoms are predicted by mild head injury among normal adolescents. J Int Neuropsychol Soc 1995; 1:121.

40 Marin RS, Biedrzycki RC, Firinciogullari S. Reliability and validity of the Apathy Evaluation Scale. Psychiatry Res, 1991;38:143-62.

41 Davis F. Empathy: A Social Psychological Approach 1994

42 Welsh KA, Butters N, Hughes J, et al. Detection of abnormal memory decline in mild cases of Alzheimer's disease using CERAD neuropsychological measures. Arch Neurol 1991:48:278-81.

43 Wood JN, Romero SG, Makale M, et al. Category-specific representations of social and nonsocial knowledge in the human prefrontal cortex. J Cogn Neurosci 2003;15:236-48.

44 Perry R, Hodges J. Differentiating frontal and temporal variant frontotemporal dementia from Alzheimer's disease. Neurology 2000;54:2277-84.

45 Rankin KP, Kramer JH, Mychack P, et al. Double dissociation of social functioning in frontotemporal dementia. Neurology 2003;60:266-71.

46 Eslinger PJ, Geder L. Behavioral and emotional changes after focal frontal lobe damage. In: Bogousslavsky J, Cummings JL, eds. Behavior and Mood Disorders in Focal Brain Lesions. Cambridge: Cambridge University Press, 2000:217-60. 